\title{
Pandemic Opens Opportunities to Solve Pathology Manpower Challenges Worldwide
}

\author{
Lewis A. Hassell* and Anoshia Afzal \\ Department of Pathology, University of Oklahoma Health Sciences Center, Oklahoma City, OK, United States
}

\section{OPEN ACCESS}

Edited by:

Mohammed S. Razzaque, Lake Erie College of Osteopathic Medicine, United States

Reviewed by: James Olumide Olufowote, University of Oklahoma, United States Anna Sarah Erem, Saba University School of Medicine, Netherlands

*Correspondence:

Lewis A. Hassell lewis-hassel/@ouhsc.edu

Specialty section:

This article was submitted to Health Communication,

a section of the journal Frontiers in Communication

Received: 29 December 2020 Accepted: 05 February 2021

Published: 17 March 2021

Citation:

Hassell LA and Afzal A (2021) Pandemic Opens Opportunities to Solve Pathology Manpower

Challenges Worldwide.

Front. Commun. 6:647345 doi: 10.3389/fcomm.2021.647345
Conventional processes of pathology education have been disrupted by the pandemic of 2020, forcing a re-evaluation of the means of teacher-learner interactions. Digital and remote teaching methods have become the standard, spearheaded by several national and international organizations and enforced by a need to social distance during a pandemic. At the same time, the public health predictions regarding the impact of non-communicable diseases on the developing world, and the resultant increased demand for pathology services to help cope with that tidal wave of disease, presents a challenge to a declining population of pathologists in North America. However, the shift in teaching methods has produced a wealth of on-line training resources. This offers for the first time a leveraging effect on the teaching value of pathology expertise, largely concentrated in the developed world, and an opportunity to both enhance and equitize training internationally, potentially sufficient to meet the rising wave of noncommunicable diseases ahead in the next 20 years. We propose four next steps to take advantage of the current opportunity to meet this challenge: Curate and organize digital training materials; Invest in the digital pathology infrastructure for education and clinical care; Expand student exposure to pathology through virtual or in person electives; Develop further competency-based rather than time-based certification and board eligibility.

Keywords: virtual pathology education, pathology manpower, low-resource settings, cancer prevalence, digital pathology

\section{BACKGROUND}

The graduate pathology education pathway for the vast extent of the 20th Century rested on a traditional model relying upon several fundamental methods and assumptions. Among these were that learning was enhanced by personal exposure to experts and to significant quantities of real and varied clinical materials. Thus learning pathology could then only happen in fairly intimate mentormentee relationships with sufficient long-term exposure to ensure adequate transmission of knowledge, habits and methods. Inasmuch as it was improved by extensive exposure to sufficient quantities of clinical materials (cases) the volume and scope of cases became a relative advantage or disadvantage in the allure of various training programs or settings. Large volume, referral centers with a diversity of case types were optimal to ensure that trainees had sufficient exposure. While the adage "see one, do one, teach one" repeated often in some disciples implied a fairly limited requisite range of exposure, in pathology the nuances of individual variation seemed to require a far broader exposure to diseases and variations to ensure an adequate core of diagnostic 
competency. The $10,000 \mathrm{~h}$ rule described and espoused in Malcolm Gladwell's book Outliers: The Story of Success seemed apt to pathology's breadth and diversity, and could even apply to individual disciplines among the progressive sub-specialization trend that occurred in the latter half of the century (Gladwell, 2009).

Likewise, undergraduate pathology education had for many decades been riveted to basic science lecture courses within the foundational years of medical school. These were commonly supplemented by gross and microscopy-focused laboratory sessions intended to teach foundational principles like wound repair, inflammation and neoplasia that had broad application in medical practice. The barriers to learning were primarily time and resource based, such as the amount of time required to surmount the learning curve of operating a microscope, and the limitations imposed by glass slide sets that were subject to age, damage and inconsistencies of content. Adequate instructional attention by professors and laboratory assistants could also be a relative barrier in some programs. In many resource-limited settings, access to quality, functional microscopes and high quality (or even any) glass slides imposed additional impediments for learners. Access to current texts, up to date scientific journals and experienced specialists were also problems not easily overcome in these settings.

But then the world suddenly became flat, or at least flatter (Friedman, 2005). Undersea fiber optic cables linked the continents; data transmission costs dropped, software for file sharing and crowdsourcing of content, disrupted normal channels of content distribution and quite quickly it became easy and inexpensive to search for answers, collaborate with peers or teams, make social and educational connections, attend massive on-line courses, or even to sit at the virtual microscope with a professor many time-zones or continents away. This tectonic shift in how we interact has changed the assumptions about the global supply chain of goods and services in many if not all disciplines, but perhaps none more profoundly than the content-intensive, and image-rich field of pathology practice, and thereby also pathology education. This paper intends to map some of the changes to date, and to project forward how some of the problems in medical education and public health ahead may be impacted by these changes in pathology in particular.

\section{THE PROBLEM(S) AHEAD}

The WHO and other public health organizations worldwide have been successful in turning the tide on many of the world's great nemeses, the communicable diseases, even if we are currently dealing with yet another new threat from that front. But this advance has rolled away the barriers to a potentially more devastating tidal wave of non-communicable diseases. Conservative estimates from the International Agency for Research on Cancer (IARC) place the worldwide cancer incidence in the year 2040 at over 27 million new cases per year, an increase of $62 \%$ over 2018 (not accounting for aging, tobacco use and other pro-carcinogenic factors). The distribution of these cases will moreover be very unevenly divided in the world, tending heavily toward the developing world. Since the capacity to diagnose, treat and manage the care of this tidal wave of patients is currently very limited, the potential for dire economic, social and political devastation on world populations is enormous. Foundational to any ability to confront and cope with this deluge of new cancer cases (or for that matter, diabetes and heart disease) is the ability to render an accurate and timely diagnosis and provide accurate, actionable follow-up data. However, we are yet a long way from having capable and adequately trained pathologists manning the front lines to cope with either the current needs much less any wave of cancer that may be ahead.

Efforts to build regional and thus "global" capacity in laboratory diagnosis have been undertaken in many countries (Olmsted et al., 2010). For example, the US President's Emergency Plan for AIDS Relief (PEPFAR), a program providing assistance to develop capacity to manage and diagnose HIV-related diseases in the African sub-continent, has returned favorable results and is now working to expand on that to deal with a broader range of diseases, including cancer. Many of these programs, however, suffer from the problems recounted in The Prosperity Paradox such as brain-drain, nonsustainability, and insufficient infrastructure (Christensen et al., 2019). Less ambitious projects often struggle with resources, human and otherwise, as well as funding. A sister-country collaboration between Vietnam and Laos to develop capacity in the Lao Cancer Center has seen the exchange of expertise and training, but struggles to gain traction due to an overall lack of trained physicians and a sustainable economic model that can attract (and retain) physicians, both oncologists and pathologists, to the fray (Tran Van Vinh, MD, Hospital 108, Hanoi, personal communication).

This brings us to a second feeder current for the potentially disastrous storm ahead--diagnostic manpower, particularly when it comes to the subspecialty expertise needed to optimally guide the personalized care paradigm we'll discuss below. A study published by the College of American Pathologists (CAP) in 2013 illustrates this nicely (Robboy et al., 2013). That work evaluated the demographics of the current US pathologist workforce using predictive methods based on prior history to project the number of pathologists who were likely to leave practice over the ensuing decades and project similarly the number of new pathologists who would enter the workforce. The projected deficits resulted in a significant predicted decline in available pathologists that would progressively worsen over the decade beginning in 2015. Although some other factors in pathologist workforce, such as the influence of economic shifts on retirements, the improved efficiencies from digital pathology or other technologies, or from shifts in techniques for monitoring and detecting disease (such as liquid biopsies for example) were not taken into account, the predicted increase in vacant positions has largely proven to be true (Metter et al., 2019).

Worldwide, less is known about the demographics and replacement or growth capabilities of training programs. It is evident that subspecialty trained pathologists are heavily concentrated in the US, and that the supply of capable 
pathologists is enormously deficient in broad swaths of the world. The majority of the low or middle-income countries have a ratio of pathologists: population that is orders of magnitude less than that in the United States or United Kingdom. This dilemma underscores the need to dramatically ramp-up the training capabilities while ensuring that the working environments are similarly prepared to employ and hold onto these trainees. But as illustrated in many countries by the enlistment of public health workers to do contact tracing and quarantine related tasks, such development and training of a workforce once the need is upon us is fraught with challenges (WHO, 2020; Hassell et al., 2021).

Hence the situation may be described as another "perfect storm" - a dramatic rising tide of diseases requiring the expertise of pathologists, a stagnant or even falling population of suitably trained pathologists who can offer training, facing off with an education model based on time-based multifaceted, fixed resource-intensive training in largely face to face settings with frequent mentoring direct interactions.

\section{TRENDS IN EDUCATION AND PATIENT CARE}

Several key trends have been gaining speed in pathology education, both at the undergraduate and graduate levels over the past few decades. These have been accompanied by several key trends in patient care that also dramatically impact the need for, and the demands upon pathology education (Christensen et al., 2009; Hassell et al., 2011).

The first of the educational trends (accompanied in a lagging manner on the diagnostic side) has been a move toward digitalization of images and data (Yin et al., 2016; Hassell, 2019). One hallmark of this trend is the dismantling of fixed microscopy labs and glass slide sets for undergraduate medical students, enabled initially by the digital slide box project piloted by Fred Dee at the University of Iowa and replicated in dental, veterinary, and numerous other health science settings (Dee, 2009; Rodrigues-Fernandes et al., 2020). A parallel trend is the creation of digital textbooks and image or slide galleries available through the World Wide Web, now typified by resources such as PathPresenter and the Digital Anatomic Pathology Academy from the Digital Pathology Association (DPA). Whereas a visitor to a pathology lab in Vietnam in 1995 might be welcomed if offering as a gift a cast-off, out-of-date edition of Anderson's Textbook of Pathology, a visitor to the same lab 15 years later would be astounded to find colleagues and students there bringing up quickly on their tablets or smartphone the latest journal article on a topic or pertinent illustrations from the WHO classification just published. Having all the world's library materials essentially in one's pocket flattens and shrinks the world immensely (Rodrigues-Fernandes et al., 2020).

This same microchip and related technology megatrend has fostered the migration of many previously highly complex laboratory tests to seemingly simple bedside devices that minimally trained personnel can perform and interpret. This has leveled much of the playing field in clinical laboratory sciences in some ways, though such devices have not come with the benefit of lower costs per test that might have completely disrupted care paradigms. However, simultaneously, we have witnessed the drive to highly individualized treatment for many diseases such as cancer, requiring ever increasing levels of genetic and epigenetic profiling, testing which has also migrated away from many traditional clinical laboratories into esoteric centralized high volume settings, often away from the training programs providing pathology training.

For a student on the medical education track or the pathology specialty path in 2020 , it has been a roller coaster ride to obtain a comprehensive educational experience. Starting with conventional on-site and hands-on methods and then experiencing an accelerated shift almost entirely to on-line virtual settings due to sudden response to a pandemic has been unsettling for many. Just as clinical encounters migrated to telemedical settings quite dramatically, often leaving medical students or trainees out of the loop, pathology training programs had to navigate carrying out case review with residents, a core component of training, almost entirely in a virtual setting or not at all (PathElective, 2020). And while most undergraduate digital slide lab sessions could continue without restrictions, any live lectures and many group, seated-round-the-microscope sessions had to migrate to being in front of the computer screen remotely connected. While both in-person and online/virtual options have their own benefits the lack of a coherent roadmap between the two has been challenging. Fortunately a robust response of educators and organizations, quickly created many opportunities online with few or no boundaries as to how much and how fast one could learn (Mukhopadhyay et al., 2020; PathElective, 2020). This developing digital world of training has enormous potential if we learn to organize and utilize it properly. That is still a big if when it comes to attaining competency to practice and certifying such to licensing boards or credentialing institutions.

For medical students, and to a certain degree trainees in disciplines such as orthopedic surgery, neurology and otorhinolaryngology where deeper knowledge of pathology is highly valued, the limitations on patient and faculty attending contact of the recent pandemic experience, highlights the value in pathology of virtual clerkships. With the creation of some of the first of these (pathelective.com) not only students locked out of hospital contact by the pandemic, but also students restricted from access due to distance, finances, or other barriers could deepen their knowledge in a variety of areas of pathology and laboratory practice (PathElective, 2020). Thus equipped, they can make better decisions on laboratory utilization, consultation, patient care, and specialty choice. Making such virtual electives widely available at low or no cost could also blunt some of the winds of the storm brewing as noted above by helping more students become familiar with the work of pathologists and amenable to following that career choice. This is also a key "rebranding" opportunity for pathology to raise awareness generally of a core, vibrant discipline that has been somewhat marginalized by systems-based medical curricula focused on clinical content. 
As more remote platforms are becoming available such as these elective opportunities, videotaped didactic lectures, pathology Twitter feeds, pathology-oriented FaceBook groups and online teaching slide sessions, the critical need becomes how to organize and promote these platforms such that the very limited boundary restrictions they offer (no or low tuition, no geographic restrictions, etc.) can work to solve meaningfully the challenges ahead, rather than just be a stop-gap filler for this current crisis (Mukhopadhyay et al., 2020; Weinstein et al., 2009; PathElective, 2020; Rodrigues-Fernandes et al., 2020). While examples of self-organizing educational teams exist, as described by Sugata Mitra in his TedTalk about the children who organized themselves to learn English and coding in order to use the free computer terminals left in their low-income neighborhood (Mitra 2010), to expect a potential physician or pathologist to scour the myriad resources and selectively find those that can fill the sufficiency of their current and future needs online seems an unreasonable ask. And to expect that selfdirected study to lead to any functional sort of degree or certificate is imprudent. But a medical school or residency training program "in the cloud" without walls, might not be such a pipe dream given what we are learning from the pandemic.

We will discuss below more of the particulars and action steps that may enable these established trends, and the catalyzing impact of the COVID19 pandemic to truly transform medical and pathology education in a manner that will empower the people of the world to meet the challenges we have already described and which loom ahead.

But one other trend in patient care deserves mention before we move on to the next section. The personalization of medical diagnosis and treatment has devolved upon an increasing array of highly complex testing, which by necessity can only be performed in specialized reference laboratories. This has made it difficult for trainees in sites without direct access to such a lab to learn key features about the development, quality control, management and performance of those tests, skills and knowledge that may be required for competency as a practicing pathologist. Currently there is little in the way of virtual training that can bridge the gap in those areas either by video conferences, problem simulations, case studies or other materials. In parallel, but opposite manner, less complex testing has moved out of the laboratory to the patient bedside as point of care devices have proliferated allowing shorter information loops and fostering or accelerating care, as we have noted above. But this testing may not always be within the purview of laboratory direction and hence within the curriculum of trainees, again creating a potential gap. It therefore becomes more and more important to digitize and standardize the training options to bridge all these deficits smoothly. To make great future professionals, it is necessary to prepare current trainees according to the dynamic nature of medicine or pathology. Some fine examples of solutions to similar curriculum gap issues are programs such as the "Laboratory Management University" (LMU) produced by the American Society of Clinical Pathology (ASCP) and American Pathology Foundation (APF), and the "University of Pathology Informatics" a joint effort of the ASCP and the Association for Pathology Informatics (API), both of which have been developed and deployed over the past 10 years in a virtual format. In the case of LMU, over $50 \%$ of pathology training programs in the US have made its contents available to their trainees, while laboratory technologists have also made use of its contents. The wave of first "graduates" of the core content came in 2013, prompting the developers to develop an advanced level curriculum including significant thesis-type projects incorporating their learnings and helping to polish management skills (Ronald Weiss MD, personal communication.) More such collaborations are needed to fill other gaps.

The challenges imposed upon pathology due to a pandemic forced adaptation so as not to compromise education and training. For the trainees, this was a whole new experience with little or no access to hands-on training, relying solely on a digital world and virtual techniques for learning purposes. For many of these "digital natives", this hasn't been bad as they quickly adapted to the new virtual "reality" of their educational world. For some the changes may have even accelerated their learning due to a reduction in non-learning activities. To the educators however, the abrupt transition to virtual teaching using digital tools was fraught with challenges and an immediate sense of their teaching effectiveness and quality being diminishedresults derived from a survey of faculty and program directors reported elsewhere, and detailed in Table 1 (Hassell et al., 2021).

\section{PUBLIC HEALTH TRENDS AND CHALLENGES}

In turning the corner into the decade of the 2020s, many agencies and health-concerned entities paused to reflect on goals and accomplishments in promoting health. The WHO identified 13 areas of urgent action for the next decade to improve health, among which was "investing in the people who defend our health" a move to target the underfunding of human resources in the health care sector (WHO, 2020). Similarly, the US Department of Health and Human Services in the Healthy People 2030 initiative has established numerous target goals in a wide array of components of and contributors to health (Healthy People 2030, 2020). Needless to say, numerous goals within the Healthy People 2030 framework have implications for laboratories and pathologists. Additionally, the payment systems within healthcare in the United States are undergoing flux, shifting emphasis from volume of testing to value in outcomes. While one might assume that this shift of rewards would reduce demand on pathologists, in reality, the demand may be increased, as in addition to their current workload under the volume paradigm, they will increasingly see demands for their expertise in rendering population health assistance and management. This is directly due to their proximity to and often stewardship for the vast trove of patient testing information that will drive effective outcomes management and improvements within the public health initiatives such as Healthy People 2030.

Implicit in this public health shift toward rewarding value- and outcomes-driven care is the dependence upon large streams of 
TABLE 1 | Potential long term pros and cons of virtual learning for pathology.

\section{Potential benefits of}

virtual training for

students and trainees

More student autonomy (choice of where, when, how and what to study) Increased variety of curriculum options and modalities available to choose from

Short-segment learning enabled via social media platforms like FaceBook, Twitter and Youtube

Performance and time spent on cases can be tracked digitally.

Exposure to multiple institutes and a global community of teachers and learners fosters seeing differing approaches to different cases.

Lower costs due to reduced travel, reduced physical facilities space and equipment, and leveraged instructional effort spread over many learners.

Low-volume, rare event situations can be made available to a wider audience for extended educational impact

Searchable database of instructional options can further extend learning

\section{Disadvantages, unexpected, or}

unintended adverse outcomes
Technological breakdowns create a vulnerability

Sham attendance or other forms of non-engagement become common; "multitasking" during educational sessions becomes much easier potentially creating bad habits

Assessments and competency forced into the digital/virtual realm become more difficult to validate

Transfer of live, hands-on experience to virtual training in areas like blood bank, microbiology and chemistry where benchwork is necessary to learn becomes problematic.

Immersion in a virtual learning environment may make students and residents reluctant to perform on-site tasks which pose risk or seem "non-essential" to training.

Excess screen time and reduced physical activity can be bad for vision, posture and overall health, potentially leading to problems like obesity, backaches etc.

Clinical encounters and practical experience like communicating with patients,

surgeons and laboratory colleagues is reduced and interpersonal skills thus not honed. patient data. There is little mystery to the moguls of modern information technology (be they Google, Amazon or others) that there is power in the data streams gathered from even seemingly trivial choices like searches for COVID-19 symptoms (StephensDavidowitz, 2020), or purchases of anti-inflammatory medications. Harnessing this information for the public good may be one of the coming century's great challenges. But it is clear that early responses to detectable shifts in such data, either from individuals or populations, will become a key tool in our ability to reach ambitious public health goals, whether that is reduction in obesity and diabetes, early detection of cancer or heart disease, or tracking an emerging infection.

Informatics has become an increasingly important component of pathology education and training. Recognized as a distinct subspecialty with its own certifying examination (offered to candidates completing core materials from several disciplines including pathology) in 2015, the understanding and management of data, computer technology, data sets, and other digital tools have also become a core area of competency required of every pathology trainee. This imperative coincides well with the trends noted above. But again, not all training settings are ideally equipped to provide these kinds of training experiences. The COVID-19 pandemic exposed this weakness as many institutions struggled to adapt to remote sign-out options using digital pathology (Hassell et al., 2021).

Telehealth has taken a dramatic leap forward during the pandemic, and continues to hold great potential to flatten the world in terms of access to care for remote populations. This trend holds true for pathology as well. Telepathology refers to the practice of pathology from a distance via image or data-sharing systems (Tran and Hassell, 2020). Primary diagnosis as well as quality control, peer review or secondary consultation can readily be performed in this mode. Several of the projects around the world aimed at improving capacity and quality of care have exploited telepathology in a variety of forms. The ASCP focus on African laboratory capacity has provided telepathology support for example (Fleming et al., 2016). Even before that, streaming video consultation has been used effectively by individuals such as Dr. Thuy Phung of Texas Children's Hospital, in teaching and supporting dermatopathology in Ho Chi Minh City, or by Dr. Matt Zarka of the Mayo Clinic in cytopathology in several locations, and by others. Fixed image telepathology has been used by the author to provide diagnostic support to a referral laboratory in Hanoi since 1995.

Digital pathology is now increasingly viewed as an enabling technology (Weinstein et al., 1987). Just as digitization of radiologic materials in the late 1990s opened the door for development and deployment of computer-assisted diagnostic tools, a host of similar efforts are underway in pathology.

Automated, FDA-approved algorithms already exist for evaluation of quantitation of staining characteristics for breast prognostic markers, and some other applications (Lloyd et al., 2010). Several venture-funded companies as well as many individuals and institutions are working on additional tools to augment the capability of pathologists to do their work using the various deep learning, artificial intelligence capabilities of fast computers and digitized images. Specific strengths of such tools can include rare-event detection, quantitation, quality control verification, and potentially image/pattern comparison to expand or narrow differential diagnostic considerations, again mirroring the path of such tools in radiology. Stand-alone functionality to replace human interaction or decision will probably face a very stiff path to approval, whereas adjunctive functions to enhance pathologist performance should be readily adopted and more easily approved. While use of these kinds of tools does not require in-depth understanding of coding or computer hardware necessarily, any more than a nurse needs to understand the solidstate chemistry on a urine dip-stick test used in an outpatient setting, nevertheless, the greater the complexity of the situation encountered mandates quality and safety controls that arise out of individuals with deeper understanding of the diseases, the technology and the various potential pitfalls involved.

These trends underline the importance of on-going training for faculty and current practitioners as well as trainees to enable 
them to teach and practice in the world today and the world ahead.

Finally, it is important to mention the regulatory and social environment in which all of these changes are happening. In almost all political domains, the practice of medicine is governed by a licensing process that requires educational credentials. Such licensing boards operate within a legal construct that specifies a scope of practice. Additional credentialing scrutiny is often performed by hospitals, practices or laboratories, accrediting agencies, specialty certifying boards and insurers or other payment systems. In most settings these processes are transparent, perhaps even public information, and intended to protect the health of the population. However, in some circumstances, they may also operate out of other interests, as a proprietary guild, to impose barriers to entry for economic or other reasons more than public safety. Disruptions to the status quo, even if safe to the public and effective for greater health, may not easily overcome these barriers (Christensen et al., 2009).

One major and truly public health challenge lies in the decision to invest in the infrastructure to implement costly new technologies such as digital pathology. Often with digital pathology, if one is to convince professionals to make practice changes and to convince the management and administration of a hospital or clinic to fund the needed purchases, the evaluation of costs expended relative to benefits obtained must be widened to the system or society level. This is due to the great asymmetry in those costs and benefits. In other words, costs tend to be local and immediate, while the benefits are often delayed or long term and more widely spread across stakeholders. If digital pathology is to be an enabling shift for pathology education and practice, and thus an enabling solution to the problems noted above, health systems, government agencies, and broad-perspective individuals will be needed to guide the strategic decisions that will open that door.

One of the lessons from COVID-19 is that things can always be done by alternative means if we don't have the choice to continue conventional methods (Wobker et al., 2020). The stress imposed upon one component of the system creates an opportunity in another. The innovation and removal of barriers required by the pandemic has created a unique opportunity for pathology education and practice not likely to be repeated, but with great potential value to future generations if they can be directed to solve the even greater problems ahead. Although there are always opportunities to improve the medical and pathology education systems, the unique circumstances of the current situation would seem to allow us to change the education dynamics forever in a positive way.

\section{OPPORTUNITIES AND SOLUTIONS}

Based on our assessment of this current and anticipated future state, we have several recommendations for action that can move us closer to a solution for the vexing public health challenges noted above, and avoidance of the storm brewing that would make them worse.

First, take advantage of the sudden deluge of on-line learning activities for pathology postgraduate medical education to not only fill a temporary gap, but to systematically enhance and standardize conventional training. Organizing these materials into a systematic structure could readily be undertaken by existing leadership in pathology training such as within the Association of Pathology Chairs Program Directors group, or other entities like the American Board of Pathology, or International Academy of Pathology. These should then be coupled with competency assessments to both affirm to the learner their progress, but also to attest to training programs and certifying boards the capacity and competency. Maintaining a low cost structure for all these activities is especially desirable to expand the reach and impact of these programs into the developing world, where quality of post-graduate training is even more uneven. Integration of such materials into on-line courses, interactive platforms, or into a structure such as the Khan Academy could then become the "Pathology Residency in the Cloud" offering some form of certification that could lead to a degree of functionality. Ideally, this could be linked to willing physical sites to provide polishing of manual skills, interpersonal skills not otherwise well assessed, and validation of the on-line assessments. It would be especially helpful if this process were directed toward potential trainees in the most underserved regions of the world. Organizations or institutions can foster this kind of mentoring, to the benefit of both mentors and mentees. This flattening effect will help prepare the world for the deluge of non-communicable diseases ahead that will require competent pathologists to be in place ahead of time.

Of course, such a change in diagnostic resources cannot occur in a vacuum. As Christensen's work has nicely demonstrated with several historical examples, endeavors that "pull" supporting resources into underserved areas, the consequences of innovation, consistently work better than efforts to "push" them in from above or outside (Christensen et al., 2019). However, one means that can facilitate this concept of pulling in resources are effective health communications on key priorities like cancer prevention (Best et al., 2017). The means of using public health messaging, or a health literacy effort to enhance the training and retention of providers in a low resource setting is a worthy topic for further publications.

Second, public health oriented non-governmental organizations, as well as health systems, academic institutions and other stakeholders should prioritize funding to invest in digital pathology infrastructure that can link areas of need with areas of capacity, and support the enhancement of digital educational and practice tools such as telemedicine, that can help to flatten the misdistribution of specialty expertise and disease distribution. Such investment may yield additional benefits to society in the form of digital data streams that can assist with disease tracking, diagnosis, prevention and optimal management.

Thirdly, existing medical schools could inform and encourage students to use one or more of the virtual pathology resources to increase their understanding of the optimal use of laboratories, and gain insight into the role of pathologists in the healthcare team by participating in one or more virtual electives during their training (Mukhopadhyay et al., 2020; PathElective, 2020). This would have the impact of directing suited students into this specialty more 
efficiently, rather than as a belated discovery after a year or more in other training programs. It would also help raise the profile of pathology as a viable, key clinical discipline as noted above.

Fourthly, the move toward the full use of competency assessments to certify candidates for board examination (and hence independent practice) should continue, with a relaxation on the time-based stipulations that currently are in place in the US and other jurisdictions. This would open the door for suitably virtually certified individuals to enter training programs for a shorter, but appropriately intense, on-site training period, and thus potentially produce more certified trained pathologists, without expanding the number of funded residency positions.

These are not trivial suggestions, we acknowledge. But neither are the problems looming ahead. Creative thinking and a willingness to seize the opportunities presented by both our technologic capabilities and our social circumstances are

\section{REFERENCES}

Best, A. L., Vamos, C., Choi, S. K., Thompson, E. L., Daley, E., and Friedman, D. B. (2017). Increasing routine cancer screening among underserved populations through effective communication strategies: application of a health literacy framework. J Cancer Educ. 32, 213-217. doi:10.1007/s13187-017-1194-7

Christensen, C. M., Grossman, J. H., and Hwang, J. (2009). The innovator's prescription: a disruptive solution for health care. New York, NY: McGraw-Hill Companies.

Christensen, C. M., Ojomo, E., and Dillon, K. (2019). The prosperity paradox: how innovation can lift nations out of poverty. New York, NY: HarperCollins.

Dee, F. R. (2009). Virtual microscopy in pathology education. Hum. Pathol. 40 (8), 1112-1121. doi:10.1016/j.humpath.2009.04.010

Fleming, K. A., Naidoo, M., and Wilson, M. (2016). An essential pathology package for low- and middle-income countries. Am. J. Clin. Pathol. 147, 15-32. doi:10. 1093/ajcp/aqw143

Friedman, T. L. (2005). The world is flat: a brief history of the twenty-first century. New york, NY: Farrar, Straus and Giroux.

Gladwell, M. (2009). Outliers: the story of success. Harlow, England: Penguin Books.

Hassell, L. A., Fung, K. M., and Chaser, B. (2011). Digital slides and ACGME resident competencies in anatomic pathology: an altered paradigm for acquisition and assessment. J. Pathol. Inform. 2 (1), 27. doi:10.4103/21533539.82052

Hassell, L. A., Peterson, J., and Pantanowitz, L. (2021). Pushed across the digital divide: impact of COVID19 on pathology teaching and learning. Acad. Pathol. 8, 1-8. doi:10.1177/2374289521994240

Hassell, L. A. (2019). Solutions for the looming cancer wave in the developing world: moving from compassion to commerce. Clin. Oncol. 4, 1573.

Healthy People 2030 (2020). Health.gov. Available at: https://health.gov/our-work/ healthy-people-2030 (Accessed September 10, 2020).

Lloyd, M. C., Allam-Nandyala, P., Purohit, C. N., Burke, N., Coppola, D., and Bui, M. M. (2010). Using image analysis as a tool for assessment of prognostic and predictive biomarkers for breast cancer: how reliable is it? J. Pathol. Inform. 1 (1), 29. doi:10.4103/2153-3539.74186

Metter, D. M., Colgan, T. J., Leung, S. T., Timmons, C. F., and Park, J. Y. (2019). Trends in the US and Canadian pathologist workforces from 2007 to 2017. JAMA Netw. Open 2 (5), e194337. doi:10.1001/jamanetworkopen.2019.4337

Mitra, S. (2010). Kids can teach themselves. Available at https://www.ted. com/talks/sugata_mitra_kids_can_teach_themselves?utm_campaign= tedspread\&utm_medium=referral\&utm_source=tedcomshare (Accessed September 23, 2020).

Mukhopadhyay, S., Booth, A. L., and Calkins, S. M. (2020). Leveraging technology for remote learning in the era of COVID-19 and social distancing. Arch. Pathol. Lab. Med. 144 (9), 1027-1036. doi:10.5858/arpa.2020-0201-ED required at such a moment as this if we are to avoid the storms ahead which may wreak even worse havoc economically and socially than the most recent COVID-19 pandemic.

\section{AUTHOR CONTRIBUTIONS}

All authors listed have made a substantial, direct, and intellectual contribution to the work and approved it for publication.

\section{ACKNOWLEDGMENTS}

The authors gratefully acknowledge the capable secretarial and technical assistance of Rebecca Brum.

Olmsted, S. S., Moore, M., and Meili, R. C. (2010). Strengthening laboratory systems in resource-limited settings. Am. J. Clin. Pathol. 134 (3), 374-380. doi:10.1309/AJCPDQOSB7QR5GLR

PathElective (2020). Pathelective.com. Available at: https://www.pathelective.com/ (Accessed August 27, 2020).

Robboy, S. J., Weintraub, S., and Horvath, A. E. (2013). Pathologist workforce in the United States: I. Development of a predictive model to examine factors influencing supply. Arch. Pathol. Lab. Med. 137 (12), 1723-1732. doi:10.5858/ arpa.2013-0200-OA

Rodrigues-Fernandes, C. I., Speight, P. M., and Khurram, S. A. (2020). The use of digital microscopy as a teaching method for human pathology: a systematic review. Virchows Arch. 477 (4), 475-486. doi:10.1007/s00428-020-02908-3

Stephens-Davidowitz, S. (2020). Google searches can help us find emerging covid19 outbreaks. The New York times. Available at: https://www.nytimes.com/ 2020/04/05/opinion/coronavirus-google-searches.html (Accessed April 5, 2020).

Tran, B. N., and Hassell, L. A. (2020). Pathologyoutlines.com. Available at: http:// www.pathologyoutlines.com/topic/informaticstelepathology0.html (Accessed September 29, 2020).

Weinstein, R. S., Bloom, K. J., and Rozek, L. S. (1987). Telepathology and the networking of pathology diagnostic services. Arch. Pathol. Lab. Med. 111 (7), 646-652.

Weinstein, R. S., Graham, A. R., and Richter, L. C. (2009). Overview of telepathology, virtual microscopy, and whole slide imaging: prospects for the future. Hum. Pathol. 40 (8), 1057-1069. doi:10.1016/j.humpath.2009.04.006 WHO (2020). Urgent health challenges for the next decade. Available at: https:// www.who.int/news-room/photo-story/photo-story-detail/urgent-healthchallenges-for-the-next-decade (Accessed September 10, 2020).

Wobker, S. E., Fine, S., Gonzalez, R. S., Sara, J. X., Khanafshar, E., and Mirza, K. M. (2020). Excellence available everywhere: the virtual pathology grand rounds experience. Am. J. Clin. Pathol. 154, S99. doi:10.1093/ajcp/aqaa161.217

Yin, F., Han, G., and Bui, M. M. (2016). Educational value of digital whole slides accompanying published online pathology journal articles: a multi-institutional study. Arch. Pathol. Lab. Med. 140 (7), 694-697. doi:10.5858/arpa.20150366-OA

Conflict of Interest: The authors declare that the research was conducted in the absence of any commercial or financial relationships that could be construed as a potential conflict of interest.

Copyright (c) 2021 Hassell and Afzal. This is an open-access article distributed under the terms of the Creative Commons Attribution License (CC BY). The use, distribution or reproduction in other forums is permitted, provided the original author(s) and the copyright owner(s) are credited and that the original publication in this journal is cited, in accordance with accepted academic practice. No use, distribution or reproduction is permitted which does not comply with these terms. 